\title{
Blockchain Application Design and Algorithms for Traceability in Pharmaceutical Supply Chain
}

\author{
Vikram Bali, JSS Academy of Technical Education, Noida, India \\ (iD) https://orcid.org/0000-0002-2809-8455 \\ Pawan Soni, JSS Academy of Technical Education, Noida, India \\ Tejaswi Khanna, JSS Academy of Technical Education, Noida, India \\ Shivam Gupta, JSS Academy of Technical Education, Noida, India \\ (iD https://orcid.org/0000-0002-0124-3878 \\ Shivi Chauhan, JSS Academy of Technical Education, Noida, India \\ Shivani Gupta, JSS Academy of Technical Education, Noida, India
}

\begin{abstract}
Blockchain technology has garnered attention from stakeholders in many domains, including healthcare, governance, and supply chain management. In the context of healthcare, traceability of pharmaceutical drugs in a transparent yet secure manner can be made faster and efficient with blockchain. This paper presents a blockchain-based solution for traceability known as PharmaChain. The traceability is achieved with application design and algorithms which are proposed in the work. The proposed application can be developed using hyperledger fabric deployed on dockers. The chain codes are written in javascript. The pharmaceutical blockchain proposed in this work consists of manufacturer, wholesaler, retailer, and consumer. The right for registering a drug into the blockchain is granted to the manufacturers only, and the ownership transfer of the drug is stored. This paper highlights the traceability of ownership transfer of the drug and validates its origin.
\end{abstract}

\section{KEYWORDS}

Hyperledger, Permissioned Blockchain, Pharmaceutical Supply Chain, Smart Contract, Trackability

\section{INTRODUCTION}

Blockchain technology has been seen as a modern tool to instill trust in trustless systems. It is a transaction management valuable system for various applications that require transactions to be done from untrusted parties over a network. The transactions are recorded in a ledger distributed electronically among all the participating nodes of a peer-to-peer network. These transactions are generally stored as hashes and are generated using state-of-the-art cryptographic hash algorithms. The ledger is stored in a block that is almost impossible to mold and can be updated only through a consensus protocol agreed by all connected nodes in the blockchain network. Bali et al. (2021) have suggested a solution for combating drug counterfeiting by tracing the ownership transfer using blockchain technology. The first practical solution provided by blockchain technology is the 
cryptocurrency Bitcoin. According to Hileman and Rauchs (2017), the current popularity of blockchain can be attributed to a $600 \%$ growth of value since it was deployed in 2009. Researchers in the past have done a lot of work in the area of healthcare using emerging technologies like artificial intelligence, IoT, blockchain machine learning, and big data(Sharma, Banerjee, Mathur, \& Bali, 2020; Yadav, Banerjee, \& Bali, 2020; Nagar, Aggarwal, Saxena, \& Bali 2020).

This article has been divided into six sections. The first section is the introduction, which presents the basic idea about the smart contract, consensus algorithm, and key benefits provided by a blockchain. In Section 2, blockchain applications in different finance, healthcare, and supply chains have been discussed. Section 3 describes the use of blockchain in healthcare with the help of many examples. Many ongoing and development projects are presented in which the role of blockchain in drug anti-counterfeiting has been discussed. Section 4 addresses the challenges in the pharmaceutical supply chain and how blockchain technology can provide solutions to these challenges. In Section 5, a blockchain-enabled supply chain framework, named PharmaChain, and traceability systems algorithms are presented. This section also discusses significant issues and concerns that need to be addressed before adopting blockchain-based solutions in real life. Finally, the concluding remarks and limitations are mentioned in Section 6.

Blockchain is a public and private ledger that comprises various transactional data. This ledger is implemented as a series of cryptographically connected blocks stored and maintained by every node of the blockchain network, which is of the peer-to-peer kind. The integration of distributed consensus algorithms and asymmetric cryptography works as constituents for achieving data integrity, transactional consistency, non-repudiation, and authentication. It further enhances visibility and audibility. Transactions registered in the blockchain and stored in the ledger cannot be tampered with once they are validated by the authorized peers of the blockchain network. Furthermore, robustness and reliability are the main features that can establish blockchain as a highly trusted platform executed on a network of peers that do not trust each other.

A contract is a way to define transaction rules and relationships between any two parties. A smart contract is a computer protocol hard-coded to enforce the policies or terms of agreements. Automated and credible transactions can be performed without the intervention of a trusted third party by using computer codes. Business logic can be implemented via smart contracts to enforce trust in the application layer. The consensus algorithm defines the performance of blockchain systems. Bambara et al. (2018) suggested that the underlying consensus algorithms maintain the safety and efficiency of blockchain systems. These algorithms should be resilient toward node failures and also able to deal with malicious nodes efficiently.

There are some properties of blockchain that make it suitable for use in this work. The first is decentralization, which states that a single authority cannot control it. The second is consensus, which means every participant has an identical copy of the ledger, and information is added based on the majority. The new data should not conflict with the current data that is already available on the blockchain. Any participant can access the information stored on the network. The third reason for choosing blockchain is that it has secure storage and provides data integrity. The data has its ownership and privacy. It allows the sharing of data in such a manner that traceability and accountability are maintained.

Researchers have advocated that transparency of data and transaction validation are two of the essential features of blockchain. The focus here is to provide secure and transparent access to transactional data. The blockchain system shows a higher degree of accountability with these features, which helps data privacy and access controls. Digitization of physical products is an exclusive feature of blockchain, including farm-to-fork tracking and tracing the data. The process of representing physical products as digital tokens is called the tokenization of assets. The use of this technology in providing a support mechanism in the management, storage, and sharing of generated digital assets has been proposed by Leite, Albuquerque, and Pinheiro (2020). Their work highlights evidence of 
the feasibility of blockchain applications for process automation in the intelligence and investigation segment.

Decentralization, consistency, and scalability are the three properties that blockchain platforms strive to maintain. Rowley (2018) states that a blockchain system can provide only two of three properties at a time, and there will always be a tradeoff among them. For example, platforms such as Bitcoin and Ethereum are decentralized but lack effective scalability regarding the number of nodes and transaction throughput. On the other hand, Hyperledger is a system that is scalable and consistent. It can scale more than 10,000 transactions per second at the expense of decreasing decentralization.

\section{BLOCKCHAIN APPLICATIONS IN DIFFERENT AREAS}

Blockchain has many applications in the financial sector as cryptocurrencies, which have introduced blockchain to the world. The fantastic thing is that there is no mention of the word 'blockchain' in Nakamoto's (2008) Bitcoin whitepaper. On the other hand, Magyar (2017) states that it has gained popularity over financial services such as insurance, micropayments, healthcare, and governance. The authors have categorized the applications into four categories, with each class having its application domain and associated application tool or startup. Tools based on blockchain have been organized for each domain in Table 1. The data has been gathered from various review papers and researches presented by Bech and Garratt (2017), Bocek et al. (2017), Chen and Zhu (2017), Huls (2015), and Zīle, K. and Renāte (2018).

\section{Table 1. Categorization of blockchain tools}

\begin{tabular}{|l|l|l|}
\hline \multicolumn{1}{|c|}{ Category } & \multicolumn{1}{c|}{ Domain } & \multicolumn{1}{c|}{ Tool } \\
\hline \multirow{5}{*}{ Data management } & Network infrastructure & Eris, Mastercoin, ChromaWay, Nxt \\
\cline { 2 - 3 } & Content and resource distribution & Swarm \\
\cline { 2 - 3 } & Cloud storage & Storj, MaidSafe, PeerNova \\
\cline { 2 - 3 } & Data monitoring & Modum.io \\
\hline \multirow{5}{*}{ Data verification } & Photo and video proofing & Uproov \\
\cline { 2 - 3 } & Work history verification & APPII \\
\cline { 2 - 3 } & Academical certification & Sony Global Education \\
\cline { 2 - 3 } & Proof of origin & Provenance, Tierion \\
\hline \multirow{5}{*}{ Financial } & Trade finance & Barclays, Santander \\
\cline { 2 - 3 } & Currency exchange and remittance & Kraken, Bitstamp, Coinbase, BitPesa \\
\cline { 2 - 3 } & P2P payments & Codius, BitBond, BitnPlay \\
\cline { 2 - 3 } & Insurance & Insurechain \\
\hline \multirow{5}{*}{ Other } & Prediction recording & Augur, Gnosis \\
\cline { 2 - 3 } & Ridesharing & Arcade City, La'Zooz \\
\cline { 2 - 3 } & Domain name registration & Namecoin \\
\cline { 2 - 3 } & Software license validation & TBankCoin \\
\cline { 2 - 3 } & Social voting system & \\
\hline
\end{tabular}


The categories have been defined as finance, data management, and data verification. Technologies such as cloud storage and network infrastructure are required for data management applications to be developed. Likewise, verification of data can be further be viewed as provenance, academic certification, and work history certification. Blockchain-based tools to provide better access to efficient financial instruments such as P2P payments, insurance, and trade finance have been mentioned.

Blockchain is an emerging technology that is being used in many domains. Blockchain technology was first used in the financial services sector. Treleaven, Brown, and Yang (2017) and Fanning and Centers (2016) have suggested that the blockchain can be used in the subdomain of the financial sector, which includes banking, stock trading, crypto exchanges, loans, and credit, and insurance. In addition, blockchain can be used in travel and mobility services for automotive manufacturing, car leasing, aerospace, and defense (Swati and Prasad, 2018; Yang et al., 2017). Olnes and Jansen (2018) advocated using blockchain in infrastructure services for construction, real estate, and energy management. Kamilaris, Fonts, and Prenafeta-Boldó (2019) and Chung et al. (2019) have suggested using blockchain technology in agriculture and mining services. Albeanu (2017) and Chen et al. (2018) have used blockchain to verify transcripts in the education sector.

The work presented in this article is a study that can impact the supply chain of pharmaceutical drugs. Keeping that in mind, the authors give different applications of blockchain technology in supply chains. Supply chains could be domain agnostic. The idea is to view how different types of supply chains are being implemented via blockchain. In healthcare, patient record management and prescription management are two other application areas where researchers have worked. This section reviews the work done by other researchers in the supply chain domain and pharmaceutical supply chain as a subset of it. The problem statements have been formulated after studying these articles.

\subsection{Blockchain and Supply Chain}

Maouchi, Ersoy propose a blockchain-based traceability system for a supply chain, and Erkin (2018) is known as TRADE. The TRADE system applies blockchain technology to obtain transparent and trustworthy traceability in the supply chain. Participants can access all the information required to track and verify the products in the supply chain. Each participant or actor can create a transaction according to a product identifier (PID), also known as a token, for asset transfer that consists of the complete product data. Active transactions are signed digitally to maintain integrity. As a result, consumers can view the entire life cycle from its provenance to its consumption.

Khanna, Nand and Bali (2020) proposed a permissioned blockchain model for end-to-end traceability in the supply chain. The researchers perceive the whole trace of the product in the supply chain as a chain of ownership transfer transactions that have taken place at the start of the supply chain.

Babich and Hilary (2018) have classified four critical vital features of blockchain technology, enhancing coordination and integration among all the members participating in the supply chain. The key features are the following:

- Transparency

- Validation

- Tokenization

- Automation

Transparency is related to the shared ledger of information collected from different participants and sources constituting the network. In this ecosystem, transparency is maintained by providing relevant rights to participants in the shared ledger. For example, the customer may have the right to verify the medicine as per the batch ID provided. Likewise, the drug authority head can track all the drugs as per the medicine's license number or batch ID, giving transparency to the system.

Tan Christidis and Devetsikiotis (2016) suggested that the properties of immutability and consensus-based verification together enable validation of information stored and retrieved from the 
Internet of Things (IoT) devices. In the system, validation is done by providing a user interface (UI) to a user to validate the asset. It is done by keying in the batch ID of the medicine and tracking the medicines from the provenance.

Automation specifies the convenience of executing smart contracts based on validated information on the blockchain. For example, Bali, Kumar, and Gangwar (2020) have used automation for wind speed forecasting using deep learning models and have written a smart contract for it. Furthermore, when an asset gets transferred from one player to another player, a smart contract is invoked. As a result, the owner of the asset is changed automatically.

Chen (2018) introduced virtual tokens (tokenization), representing a claim on any physical asset in the real world and transactions involving them among blockchain participants. In this case, pharmaceuticals are the tokens, where the author had represented a batch of medicine as the token and allocated them a unique batch ID. Whenever the drug is transferred from one participant to another, the owner of this batch ID changes, and the token moves ahead in the system.

\section{BLOCKCHAIN AND HEALTHCARE}

\subsection{Patient Record Management}

An essential activity of the healthcare industry is managing the patient's data, including storing, sharing, and accessing the data. Efficient management of the patient's data can enhance communication and provide integrated views of a patient's medical history. However, it isn't easy to handle and requires a proper mechanism to achieve. The patient's data is susceptible and involves a factor of trust at the time of transmission. Healthcare services and data exist in different forms in many repositories. Sometimes these platforms are not compatible with each other. This incoherent system is the cause of several problems in this sector and lays the foundation for research in this domain. Sometimes healthcare professionals do not have complete access to patient's data, which hinders the succeeding process of diagnosis and therapy. Kumar, Toorang, and Bali (2020) developed web applications for better data visibility and trend forecasting. These applications further enable users to obtain a higher level of information and make better decisions. Several blockchain-based projects have been developed in healthcare to provide more efficient solutions. Table 2 shows projects that are based on blockchain technology for the healthcare industry.

Table 2. Healthcare projects implemented using blockchain technology

\begin{tabular}{|l|l|l|l|}
\hline S. No. & Project Name & \multicolumn{1}{|c|}{ Developed/Proposed by } & \multicolumn{1}{c|}{ Description } \\
\hline 1. & MedRec & Ekblaw and Azaria (2016) & $\begin{array}{l}\text { A decentralized content-management system for health } \\
\text { records }\end{array}$ \\
\hline 2. & Patientory & Mcfarlane et al. (2017) & A health information exchange (HIE) \\
\hline 3. & GemOS & Kannan and Smith (2017) & A framework to develop distributed record systems \\
\hline 4. & MedShare & Xia et al. (2017) & A medical data-sharing system in a trustless environment \\
\hline 5. & ETDB-Caltech & Ortega (2019) & A distributed public database for electron tomography \\
\hline 6. & OPU & OPU Labs (2019) & A data-sharing system for skincare industry \\
\hline 7. & MedX Protocol & Todaro et al. (2018) & A global healthcare market \\
\hline 8. & Dermonte & Mannaro et al. (2018) & A teledermatology platform developed and tested in Italy \\
\hline
\end{tabular}




\subsection{Prescription Management}

It is vital to have proper prescription management for better healthcare services in a country. However, according to Skolnick (2018), improper use of the prescription has been unbridled in recent years, leading to many stimulant crises. Therefore, many solutions based on blockchain have been proposed to overcome the improper management of prescriptions.

BlockMedx is an Ethereum-based platform capable of securely managing prescriptions, where all the medications and transactions are stored on the blockchain. Implementing the smart contract capability provided by Ethereum, the platform maintains transactions securely and immutably. Furthermore, all the participants involved in prescription management, such as doctors, pharmacies, patients, and hospitals, are connected over the blockchain network to facilitate multiparty transactions.

Project Heisenberg, developed by Diaz (2018), uses smart contracts on Ethereum to track prescriptions. This project implements a non-fungible token standard to eliminate the risk and implicit cost of pharmaceutical script fraud. Different platforms are provided to doctors, pharmacies, and patients in the process. Furthermore, different roles and rights are assigned to these participants. Hosted on an Ethereum Consortium Network, Project Heisenberg is permissioned by a custom JSON genesis file.

ScriptDrop is another platform that works for efficient medication delivery to patients. The patients do not have to visit a pharmacy to buy medicines. ScriptDrop provides access to a network that keeps track of end-to-end data. As a result, it improves drug adherence while reducing prescription abandonment. Commercial systems such as ScriptDrop enable users to maintain better healthcare with the use of blockchain technology.

\subsection{Pharmaceutical Supply Chain and Counterfeit Drug Detection}

Efficient and effective usage of supply chains is a challenge for every industry. There is a higher risk and complication in the healthcare industry than in other sectors because of its impact on a person's health. If there is an inefficient supply chain used in the healthcare industry, this can hamper a patient's health, leading to severe consequences. Thus, proper standards of the pharmaceutical supply chain need to be followed. Clauson et al. (2018) show that blockchain technology is a promising solution for improving security, safety, trackability, and reduction of falsified medicine in the medical supply chain.

Blockchain technology provides a perfect solution for trackability and traceability for pharmaceutical products. The drug information can be stored publicly to be safe, tamper-proof, and accessible by only imperative nodes. It further adds to the safety and credibility of the supply chain. Some of the solutions that have been developed or proposed using blockchain technology are the following:

1. The MediLedger Project (2017): MediLedger is the supply chain network for pharmacy products. It is a centralized permissioned blockchain for the actors that are participating in a supply chain. The main focus is on tracking and tracing products. The project allows users to trace and track the products from their origin to their consumption.

2. BlockVerify (2018): BlockVerify is the solution for transparency in the supply chain. This platform has four prominent use cases: i) pharmacy, ii) diamonds, iii) luxury items, and iv) electronics. The main aim is to verify counterfeit products, diverted products, stolen merchandise, and fraudulent transactions.

3. Ambrosus: Ambrosus is a blockchain network for pharmaceutical and food industries based on IoT technology. It provides a secure system, distributed ledgers, and databases to enhance supply chain visibility and quality assurance in the ecosystem.

4. Authentag: This platform tracks thousands of products in the supply chain by using blockchain technology, immutable systems, and advances in tokenization. Authentag works by following unique labels or tags and connecting the tags to individuals who interact with each other. It 
tracks the relationships from origination to the utilization in that supply chain, from production to disposal. Table 3 shows some projects of companies in blockchain-enabled pharmaceutical supply chain solutions.

Table 3. Blockchain-enabled supply chain solutions in healthcare

\begin{tabular}{|l|l|l|l|}
\hline $\begin{array}{c}\text { S. } \\
\text { No. }\end{array}$ & Project Name & \multicolumn{1}{|c|}{ Description } & Domain \\
\hline 1. & $\begin{array}{l}\text { MediLedger } \\
\text { Project (2017) }\end{array}$ & $\begin{array}{l}\text { A Decentralized blockchain } \\
\text { solution to fulfill the trace and track } \\
\text { governance in the pharmaceutical } \\
\text { supply chain }\end{array}$ & $\begin{array}{l}\text { A consortium of leaders from 25 pharmaceutical } \\
\text { companies }\end{array}$ \\
\hline 2 & $\begin{array}{l}\text { BlockVerify } \\
(2018)\end{array}$ & $\begin{array}{l}\text { A solution to verify counterfeiting } \\
\text { of products, diverted products, } \\
\text { stolen merchandise, and fraudulent } \\
\text { transactions. }\end{array}$ & Pharmacy, diamonds, luxury items, electronics \\
\hline 3. & $\begin{array}{l}\text { Ambrosus } \\
(2018)\end{array}$ & $\begin{array}{l}\text { An IoT network, based on } \\
\text { blockchain for supply chain targeting } \\
\text { pharmaceutical industries and food }\end{array}$ & $\begin{array}{l}\text { Supply chain optimization, logistic tracking, quality } \\
\text { assurance, anti-counterfeiting }\end{array}$ \\
\hline 4. & $\begin{array}{l}\text { Authentag } \\
\text { (2018) }\end{array}$ & $\begin{array}{l}\text { Provides distributed ledger } \\
\text { technology using blockchain to give } \\
\text { trackability and validation services } \\
\text { for the pharmaceutical supply chain }\end{array}$ & $\begin{array}{l}\text { Government, medical devices, and pharmaceutical } \\
\text { tracking }\end{array}$ \\
\hline 5. & $\begin{array}{l}\text { IEEE Pharma } \\
\text { Supply } \\
\text { Blockchain } \\
\text { Forum (2018) }\end{array}$ & $\begin{array}{l}\text { An IEEE educational podium for } \\
\text { multiple participants to initiate } \\
\text { and talk about the potential usage } \\
\text { of pharmaceutical supply chain } \\
\text { solutions using blockchain }\end{array}$ & Education \\
\hline
\end{tabular}

\section{PROBLEM FORMULATION}

\subsection{Challenges in the Pharmaceutical Supply Chain}

In a World Health Organization (WHO) survey in 2010, it was estimated that there would be more than 1 million deaths yearly due to counterfeit pharmaceuticals. The WHO estimated that $50 \%$ of the drugs for sale were bogus, and $90 \%$ of drugs purchased online were not from the country from which the websites claimed them to be. Falsified drugs are fake medicine, which may lead to dangerous consequences. Imperative nodes of the supply chain can distinguish between original or falsified drugs as they can track the pharmaceuticals from their provenance. The binding nodes can gain transparency regarding medicines, which also helps in inventory management.

Inventory management and storage as per the prescribed specification is an issue. If there is an imbalance in demand-supply chain, it may increase the mortality rate due to improper storage management. Pharmacists should take care while stocking medicines. It is seen that some of the drugs need to be kept at a specific temperature, pressure, and other parameters to maintain goodness. Currently, the responsibility for providing these specified conditions throughout the shipment falls on the manufacturers. This results in compromised accountability for the retailer and the wholesaler, and hence the transparency of the overall system is reduced.

Consumption of fake medicines has a harmful effect and may put the patient's life in danger. Finan (2018) has observed that some safety measures such as the Falsified Medicines Directive (FMD) are 
being implemented in the European Union. The FMD addresses the current pharmaceutical supply chain challenge, but security, data integrity, and cyberattack still need to be addressed.

In the blockchain network, the transactions are stored immutably as a cryptographic hash. Data integrity can be maintained in blockchain-based pharmaceutical solutions where prescription and counterfeit drugs need to be addressed. Immutability and digital signatures provide a layer of security to sensitive transactions in pharmaceutical industries. Security issues here include data breaches, corruption, unauthorized access, and cyberattacks. The FMD addresses some pharmaceutical supply chain safety measures such as obligatory safety features, a supply chain-wide logo to identify pharmacies for the legality, strict rules on importing, and strengthening the record-keeping requirement for wholesale distributors. However, it cannot detect the compromised storage conditions of pharmaceutical drugs during the travel, resulting in poor-quality medicines and may be harmful to the patient's wellbeing.

\subsection{Use of Blockchain Technology for Improving Supply Chains}

The immutable nature of blockchain ensures that no record gets altered. Nobody can put counterfeit drugs into the system. If any unauthorized person tries to tamper with the supply chain, he will not edit the system as it will be notified to all the peers unless there is a $51 \%$ attack. Everything is transparent within the blockchain, and each actor can keep a check on the items at each level if access has been granted to them.

It is possible to track the entire pharmaceutical supply chain process from a product's provenance until the end of the supply chain. This provenance is essential in predicting and forecasting a potential fault in the system. Such predictions can be performed using deep learning algorithms proposed for wind speed forecasting by Bali, Kumar, and Gangwar (2019). A blockchain solution can bring significant improvements to the pharmaceutical industry's supply chain as it has the feature of trackability by tracking down the hash code of each block. For previous transactions, the last hash block can be traced down. Blockchain assures safety and authenticity to all parties by allowing them to track a drug throughout its pharmaceutical supply chain.

At each step in the chain, smart tags or barcodes could be scanned. This data is stored onto a system (based on blockchain) in the form of a transaction block, which eventually creates and records an audit trail of the medicine's entire flow. These blocks help one to trace down the product till provenance. There could be deaths due to a lack of knowledge of temperature, pressure, and other standard parameters at which the medicine should be stored. Sensors could be a key to overcome this problem. They can be installed into the chain, and the ledger system will be keeping pressure, temperature, and other standard parameters. These sensors have been incorporated and used to forecast wind speed using Long Short-Term Memory (LSTM) and Support Vector Machines (SVM) deep learning algorithms proposed by Gangwar, Bali, and Kumar (2019). This is especially necessary for drugs requiring refrigerated storage, such as insulin, and specially created drugs with some standard storage conditions.

When a drug is delivered to the pharmacy, pharmacists and consumers can easily track it down through the supply chain if the drug has not met the standard parameters at any step in the chain as they will have full access to the supply chain. They can find whether the drug is coming from an authentic factory or planted in the middle of the system. They can also have access to other essential instructions regarding the medicine, which will be accessible while scanning the barcode or putting the hash code on the system of that package.

Many advantages of using blockchain have been suggested by Clements et al. (2017). Blockchain reduces complexity. Hence it becomes more feasible to track and trace the drugs. The distributed ledger system stores the expiration dates of the product, which can improve the distribution and helps in inventory management. This reduces the chances of error as the drugs can be verified and authenticated by the imperative nodes. It prevents any life threat to a person and brings more transparency into the system and reliability to the supply chain. Such a system will be easy to be adopted and sellable 
across the globe due to its features such as tamper-proof nature, trackability, and identification of counterfeit drugs. When the patient does not consume the fake drugs, there will be no danger to the patient's life, and the probability of curing the disease will also increase.

Regulators and drug store people can check the entry of fake medicines into the supply chain with more accuracy by using blockchain. Biometric can verify each consignment of the drug. There are checkpoints during the drug shipment where the drug's authenticity is verified by biometric, $2 \mathrm{D}$ standardized identification sweeps, or sensor innovation. As the drug is traced from inception to its consumption, the complete process becomes consistent, precise, and secure.

\section{PROPOSED SOLUTION}

In this section, the authors propose the application design of PharmaChain for implementing the pharmaceutical supply chain with the help of blockchain. The proposed algorithms and the limitations of the work are also presented in this section. In the proposed algorithm, the blockchain operator will set up the service provided by the IBM Blockchain Platform. IBM Blockchain Platform creates a Hyperledger Fabric network on a container, like Kubernetes, and makes different manufacturers, wholesalers, retailers, and consumers. The operator will deploy chain codes (smart contract) on the network and install the chain code on Hyperledger Fabric. The Node.js server uses the Fabric Software Development Kit to interact with the network running on IBM Blockchain Platform 2.0 and creates APIs for the users to interact with the system easily. The actors can update, query the blockchain ledger, and interact with the web interface. The application design of the proposed framework is shown in Figure 1.

Figure 1. Application Design of Pharmaceutical Supply Chain over Hyperledger Fabric

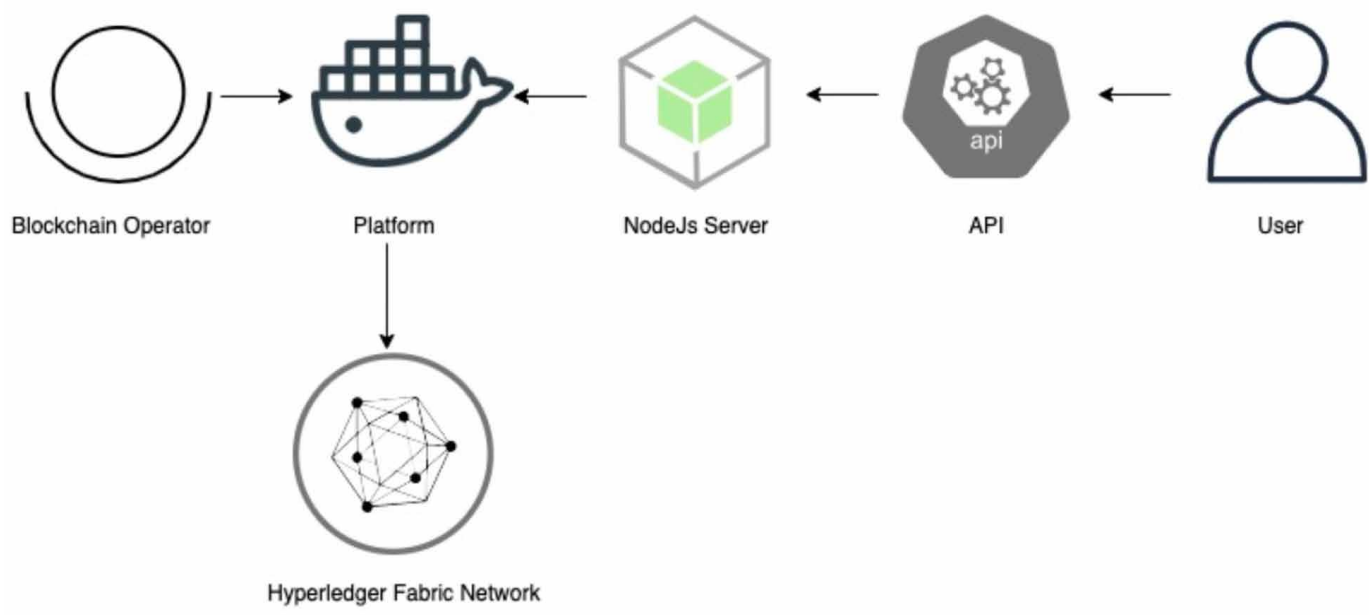

\subsection{Algorithm to Verify Traceability}

The authors have presented three algorithms to address the traceability of drugs in the pharmaceutical supply chain. The three algorithms are:

1. Add/register legitimate actors in the blockchain;

2. Add/register the drug in the blockchain; and 
3. Ownership transfer of the drug.

The following paragraphs discuss the detail of three algorithms used to verify the traceability and trackability of drugs.

1. Procedure Admin(): To add legitimate actors to the blockchain.

This is the main procedure responsible for adding actors onto the blockchain. Each actor is allocated a unique ID verified and stored at the time of any transaction onto the blockchain. This module can be initiated and executed by a pharmaceutical company. It is a centralized network where the actors first register themselves on the network, done by the admin() procedure. After being registered on the network, they can authenticate themselves with the certificates issued to them at the time of registration and participate in the transactions. The complete code for the admin() procedure is shown in Figure 2.

Figure 2. Procedure Admin()

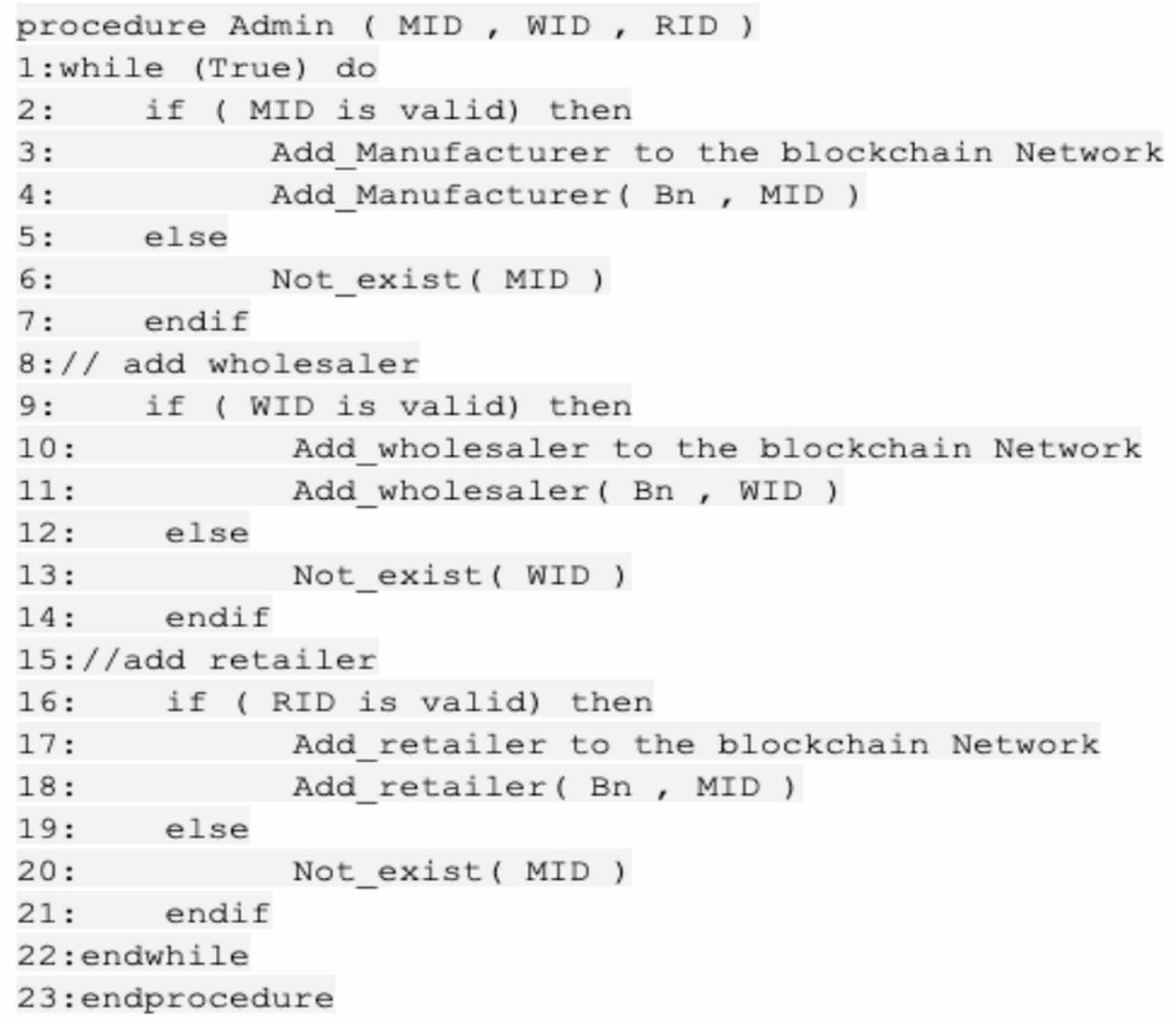


2. Procedure addDrug(): To add drug in the blockchain.

This procedure can be initiated by the manufacturer only. The manufacturer is the initial actor/ node/point of the supply chain. Thus the right to create new drugs and register them on the blockchain is granted to the manufacturer only. The first owner of the drug is the manufacturer that produces the first dot in the audit trail. The actors in the supply chain are registered and issued an authentication certificate by the procedure admin(), so there is no chance of any security breach and introduction of counterfeit drugs into the supply chain. The complete working and addition of the drug are given in Figure 3.

\section{Figure 3. Procedure addDrug()}

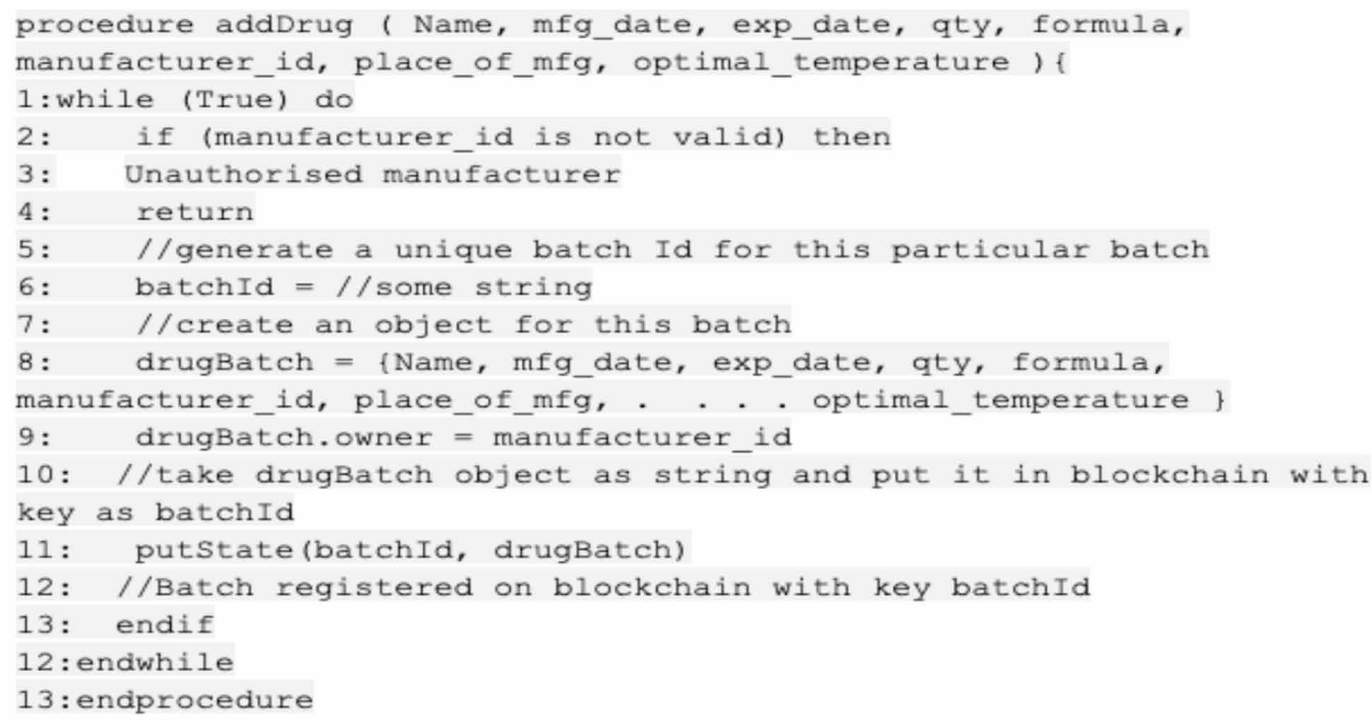

\section{Procedure ownershipTransfer(): See below.}

This procedure is invoked when drugs are transferred/moved from one actor to another. A dayto-day example of the ownership transfer of drugs is the movement of drugs from the manufacturer to the wholesaler, from wholesaler to retailer, and finally, from retailer to customer. This procedure is responsible for verifying the authenticity of the medicine or drug with the help of authentication IDs stamped on the drug when it moves down the supply chain. Each actor in the supply chain appends its unique information to the drug, which can be easily verified to identify the counterfeit medicines into the blockchain-enabled supply chain. For example, the manufacturer creates the batch_ID and appends it to the drug. If the batch_ID of the scanned medicine is not found in the blockchain, it will imply that the designated manufacturer did not manufacture the medicine. The complete code of the procedure ownership transfer is given in Figure 4.

\subsection{Transaction Flow for Traceability in Blockchain-Enabled Supply Chain}

The proposed work in this article is based on four actors in the supply chain: manufacturer, wholesaler, retailer, and customer. To authenticate and trace the journey of drugs, three procedures have been suggested in this work. The procedures are the following: 


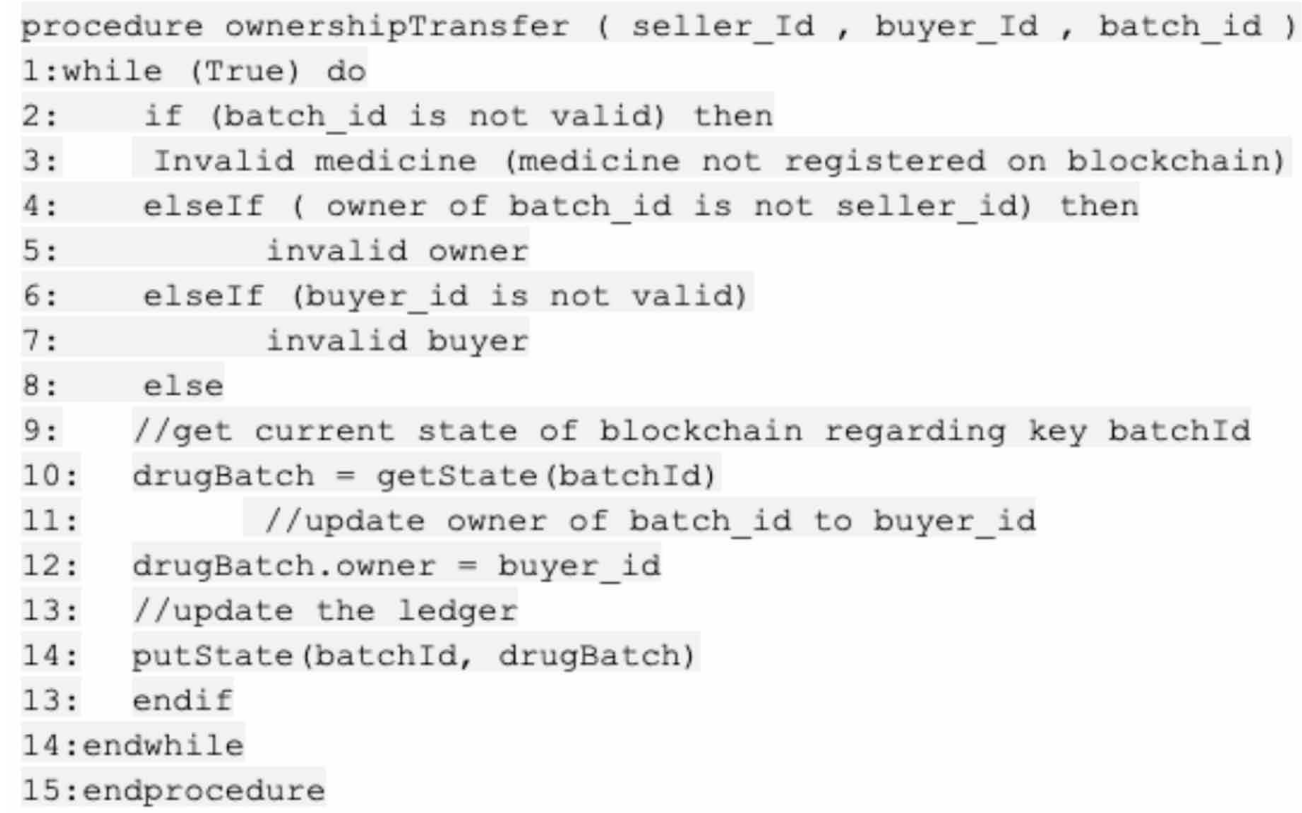

1. Add/register legitimate actors in the blockchain;

2. Add/register the drug in the blockchain; and

3. Ownership transfer of the drug.

The manufacturer is the originator of the drug and the first actor to register the drug on the blockchain ledger with a unique information that becomes the batch_ID of the drug. This batch_ID may include information of the manufacturer and data related to the medicine such as manufacturer ID, location of manufacturing unit, details of job manager, GST number, license number of the manufacturer, manufacturing date of the drug, expiry date of the drug, and composition of different salts. All this information is appended by the manufacturer using blockchain technology and makes the drug very secure to move ahead in the supply chain. When this drug is sold to a wholesaler, the wholesaler may verify the drug's authenticity by confirming the information that the manufacturer has appended. The transfer of the medicine is done through the blockchain network; therefore, there is no chance of compromising and counterfeiting the drug. After verifying the drug, the wholesaler initiates its procedure to change the ownership of the drug and adds its information to the drug and forwards it to the retailer. The retailer follows a similar mechanism followed by the wholesaler for verification and change of ownership of the drug. At the end of the supply chain, the customer receives the medication. The customer has the option of thoroughly verifying the journey of the drug from its origin. The customer becomes the last owner of the medicine and consumes it. This mechanism is bidirectionally verifiable as we can verify the journey of the drug from origin to consumption by the customer and from consumption to the source by the manufacturer. The traceability of the drug is shown in Figure 5. 


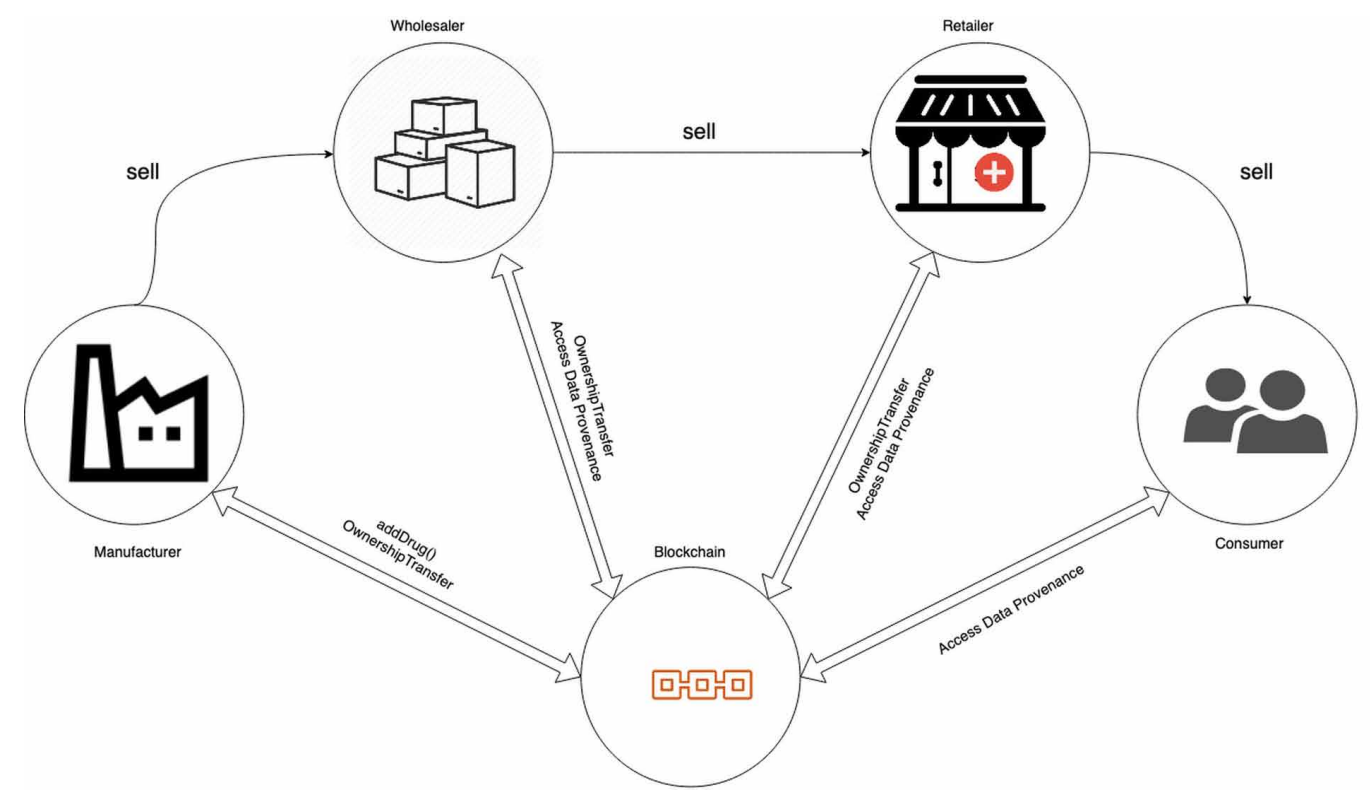

\section{CONCLUSION}

In today's era, blockchain technology can provide an effective solution to several problems, including the healthcare industry. For example, a patient-centered healthcare model with a global information exchange can be implemented by decentralized trust and incentive structures enabled by the blockchain. Similarly, vendor lock-in problems in healthcare may be resolved by the blockchain-based network.

In this article, the authors have explored and presented the applications of blockchain technology in the healthcare sector. The transaction management systems for medical prescriptions, drugs, device supply chain, treatment records, and billing need higher levels of transparency and authenticity. The authors have proposed a traceability system based on blockchain technology called PharmaChain. The algorithms including the procedures admin(), addDrug(), and ownershipTransfer() have been presented. The authors have suggested the proposed blockchain-enabled supply chain to solve traceability and counterfeit drugs in the healthcare ecosystem.

Blockchain technology has incorporated many innovative solutions in the healthcare industry. However, we can say that the application of blockchain in the healthcare industry is in its infancy. Only a few working applications with a minimal user base, whitepapers, and prototypes are available in the research domain of blockchain-based healthcare. The application of blockchain can have a significant positive impact on the healthcare industry. Adoption and migration from classical to blockchain-enabled systems involve challenges such as implementation cost, technological barriers, regulatory conformity, compatibility of devices, and scalability. These parameters need to be addressed to enable blockchain technology to create a firm footing and flourish the healthcare industry.

\subsection{Limitations}

Blockchain holds the potential to improve the healthcare system and other sectors. Many organizations have started implementing it for some specific use cases. Several challenges should be discussed before its usage in day-to-day life. In this section, some of the drawbacks of blockchain technology have been discussed. 
1. Scalability: The network must have a high transaction throughput and be scalable for blockchainbased solutions in real life. Both the conditions can be satisfied if a permissioned blockchain is used. However, in public blockchain networks, such as Bitcoin and Ethereum, scalability is a problem.

2. Interoperability and integration with the conventional systems: Blockchain technology should be able to integrate effectively and efficiently with existing devices and systems. Interoperability makes this process very challenging. Healthcare has a variety of devices that makes it even more complex.

3. Adoption and incentives for participation in blockchain: To use blockchain technology in the healthcare space, multiple stakeholders need to coordinate and cooperate. These stakeholders can include hospitals, manufacturers, pharmacists, etc. It is a human tendency to expect something in return for change management and shift to a new environment. A proper incentive scheme should be introduced for all stakeholders to fulfill these expectations.

4. Uncertainty in cost of operation: It is challenging to estimate the operational cost of a blockchain network. A significant number of resources are already being used in the healthcare sector. There is always a need of updating and troubleshooting the system in case of errors. It is better if the cost of the blockchain-based approach can be calculated more accurately beforehand.

The work suggested in this article discusses how to overcome the scalability problem as there can be any number of actors in the healthcare supply chain scenario depicted in this work. The network will have a high transactional rate because it will be developed over a permissioned blockchain. Interoperability and integration are possible for organizations that are newly established in the area of healthcare. The freshly installed manufacturing units will have high-end devices that are compatible with blockchain-enabled devices. The work presented in the article also discusses the limitation for participation in blockchain as all the stakeholders, that is, manufacturer, wholesaler, retailer, and customer, can verify the origin of the drug and its authenticity. Therefore, the incentives for all the actors are the delivery of the drug in good condition. The work presented in this research does not mention the estimated cost for the implementation of blockchain. Estimating the implementation cost of blockchain lies in the domain of software estimation, network management, and technology implementation. These three domains have to be studied in depth for calculating the cost of a blockchain-enabled network, which is the prime limitation of this study and paves the path for future work. The proposed PharmaChain framework is a simplified picture of the actual market scenario involving only four actors, whereas, in reality, the number of actors is higher. A hybrid framework should be studied in future work based on all the actors involved in the supply chain. 


\section{REFERENCES}

Albeanu, G. 2017. Blockchain technology and education. The 12th International Conference on Virtual Learning, 271-275. doi:10.1504/IJCSE.2020.109403

Authentag. (2018). Available: https://www.authentag.com/

Babich, V., \& Hilary, G. (2018). Distributed ledgers and operations: What operations management researchers should know about blockchain technology. Manufacturing \& Service Operations Management; Georgetown McDonough School of Business Research Paper No. 3131250. 10.2139/ssrn.3131250

Bali, V., Khanna, T., Soni, P., Gupta, S., Chauhan, S., \& Gupta, S. (2021). Combating drug counterfeiting by tracing ownership transfer using blockchain technology. International Journal of E-Health and Medical Communications, 13(3), 1 .

Bali, V., Kumar, A., \& Gangwar, S. (2019). Deep learning-based wind speed forecasting- A review. In International Conference, Confluence on Cloud Computing, Data Science \& Engineering. Amity University Noida. doi:10.1109/ CONFLUENCE.2019.8776923

Bali, V., Kumar, A., \& Gangwar, S. (2020). A novel approach for wind speed forecasting using LSTM-ARIMA deep learning models. International Journal of Agricultural and Environmental Information Systems, 11(3), 13-30. doi:10.4018/IJAEIS.2020070102

Bambara, J. J., Allen, P. R., Iyer, K., Lederer, S., Madsen, R., \& Wuehler, M. (2018). Blockchain: A practical guide to developing business, law, and technology solutions. McGraw Hill Professional. https://www.oreilly. com/library/view/blockchain-a-practical/9781260115864/

Bech, M. L., \& Garratt, R. (2017, Sept.). Central Bank Cryptocurrencies. BIS Quarterly Review. Available: https://www.bis.org/publ/qtrpdf/r_qt1709f.htm

Behner, P., Pisani, J., Ehrhardt, M., Edmunds, R., Fricker, R., \& Hotz, D. (2017). Fighting counterfeit pharmaceuticals: New defences for an underestimated and growing menace. PwC Strategy. Available: https:// www.strategyand.pwc.com/gx/en/reports/fighting-counterfeit-pharmaceuticals.pdf

Block Verify Blockchain-Based Anti-Counterfeiting. (2018). Available: http://www.blockverify.org/

BlockMedx - Secure E-Prescribing Platform. (2018). Available: https://blockmedx.com/

Bocek, T., Rodrigues, B., Straser, T., \& Stiller, B. (2017). Blockchains Everywhere - A Use-Case of Blockchains in the Pharma Supply-Chain. IFIP/IEEE Symposium on Integrated Network and Service Management, $772-777$. doi:10.23919/INM.2017.7987376

Chen, G., Xu, B., Lu, M., \& Chen, N. S. (2018). Exploring blockchain technology and its potential applications for education. Smart Learning Environments, 5(1), 1-10. doi:10.1186/s40561-017-0050-X

Chen, Y. (2018). Blockchain tokens and the potential democratization of entrepreneurship and innovation. Business Horizons, 61(4), 567-575. doi:10.1016/j.bushor.2018.03.006

Chen, Z., \& Zhu, Y. (2017). Personal Archive Service System Using Blockchain Technology: Case Study, Promising and Challenging. IEEE International Conference on AI \& Mobile Services, 93-99. doi:10.1109/ AIMS.2017.31

Christidis, K., \& Devetsikiotis, M. (2016). Blockchains and Smart Contracts for the Internet of Things. IEEE Access: Practical Innovations, Open Solutions, 4, 2292-2303. doi:10.1109/ACCESS.2016.2566339

Chung, K., Yoo, H., Choe, D., \& Jung, H. (2019). Blockchain network based topic mining process for cognitive manufacturing. Wireless Personal Communications, 105(2), 583-597. doi:10.1007/s11277-018-5979-8

Clauson, K., Breeden, E., Davidson, C., \& Mackey, T. (2018). Leveraging blockchain technology to enhance supply chain management in healthcare. Blockchain in Healthcare Today, 1. Advance online publication. doi:10.30953/bhty.v1.20

Clements, C., Cushley, S., Davies, S., \& Mone, A. (2017). Accurate, audited and secure: How Blockchain could Strengthen the pharmaceutical supply chain. Price Waterhouse Coopers LLP. https://www.pwc.co.uk/healthcare/ pdf/health-blockchain-supplychain-report\%20v4.pdf 
Craib, R., Bradway, G., \& Dunn, X. (2018). Ambrosus White paper. https://ambrosus.com/assets/en/AmbrosusWhite-Paper.pdf

Diaz, T. (2018). Project Heisenberg: Solving prescription pharmaceutical logistics using smart contracts. https:// github.com/tylerdiaz/Heisenberg

Ekblaw, A., \& Azaria, A. (2016). Medrec: Medical data management on the blockchain. Viral Communications. https://viral.media.mit.edu/pub/medrec

el Maouchi, M., Ersoy, O., \& Erkin, Z. (2018). TRADE: A transparent, decentralized traceability system for the supply chain. Proceedings of the 1st ERCIM Blockchain Workshop, Reports of the European Society for Socially Embedded Technologies. 10.18420/blockchain2018_01

Fanning, K., \& Centers, D. P. (2016). Blockchain and its coming Impact on Financial Services. Journal of Corporate Accounting \& Finance, 27(5), 53-57. doi:10.1002/jcaf.22179

Finan, T. (2018). Blockchain and the pharmaceutical supply chain: driving security and transparency. https:// aws.amazon.com/blogs/apn/blockchain-and-the-pharmaceutical-supply-chain-driving-security-and-transparency/

Gangwar, S., Bali, V., \& Kumar, A. (2019). Comparative analysis of wind speed forecasting using LSTM and SVM. EAI Endorsed Transactions on Scalable Information Systems, 25(1), 1-8. doi:10.4108/eai.13-702018.159407

Hileman, G., \& Rauchs, M. (2017). 2017 Global Blockchain Benchmarking Study. Cambridge Centre of Alternative Finance. doi:10.2139/ssrn.3040224

Huls, C. (2015). A Scenario Planning for Interbank Payments and Decentralized Ledger Platforms. Master thesis. University of Twente. http://essay.utwente.nl/66966/1/Huls_MA_EWI.pdf

Kamilaris, A., Fonts, A., \& Prenafeta-Boldú, F. X. (2019). The rise of blockchain technology in agriculture and food supply chains. Trends in Food Science \& Technology, 91, 640-652. doi:10.1016/j.tifs.2019.07.034

Kannan, S., \& Smith, M. (2017). Improving patient care coordination and outcomes using blockchains. Gemology Whitepaper. https://211hzr1wjznm2pclk01j90ly-wpengine.netdna-ssl.com/wp content/uploads/2016/10/ GemOSPlatformWhitepaper.pdf

Khanna, T., Nand, P., \& Bali, V. (2020). Permissioned blockchain model for End-to-End trackability in supply chain management. International Journal of e-Collaboration, 16(1), 45-58. doi:10.4018/JJeC.2020010104

Kumar, A., Toorang, P., \& Bali, V. (2020). User based load visualization of categorical forecasted smart meter data using LSTM network. International Journal of Multimedia Data Engineering and Management, 11(1), 30-50. doi:10.4018/IJMDEM.2020010103

Leite, G. S., Albuquerque, V. B., \& Pinheiro, P. R. (2020). Process Automation and Blockchain in Intelligence and Investigation Units: An Approach. Applied Sciences (Basel, Switzerland), 10(11), 3677. doi:10.3390/app10113677

Magyar, G. (2017). Blockchain: Solving the privacy and research availability tradeoff for EHR data: A new disruptive technology in health data management. IEEE 30th Neumann Colloquium (NC), 135-140. doi:10.1109/ NC.2017.8263269

Mannaro, K., Baralla, G., Pinna, A., \& Ibba, S. (2018). A blockchain approach applied to a teledermatology platform in the Sardinian region (Italy). Information (Basel), 9(2), 44. doi:10.3390/info9020044

Mcfarlane, C., Beer, M., Brown, J., \& Prendergast, N. (2017). Patientory: A healthcare Peer-to-Peer EMR storage network. https://patientory.com/wp-content/uploads/2017/04/Patientory_Whitepaper-1.pdf

MedX Protocol. (2018). https://medxprotocol.com

Medicalchain. (2018). Whitepaper 2.1. https://medicalchain.com/Medicalchain-Whitepaper-EN.pdf

MediLedger. (2017). Building an Open Network for the Pharmaceutical Supply Chain. https://www.mediledger. $\mathrm{com} /$

Nagar, R., Aggarwal, D., Saxena, U. R., \& Bali, V. (2020). Cancer prediction using machine learning techniques based on clinical \& non-clinical parameters. International Journal of Advanced Science and Technology, 29(4), 8281-8293. http://sersc.org/journals/index.php/IJAST/issue/view/263 
Nakamoto, S. (2008). Bitcoin: A Peer-to-Peer Electronic Cash System. https://bitcoin.org/bitcoin.pdf

Olnes, S., \& Jansen, A. 2018. Blockchain technology as infrastructure in public sector: an analytical framework. Proceedings of the 19th Annual International Conference on Digital Government Research: Governance in the Data Age, 1-10. doi:10.1145/3209281.3209293

OPU Labs. (2018). https://www.opucoin.io/wp-content/uploads/2019/07/OPU-Whitepaper-v4.pdf

Ortega, D. R., Oikonomou, C. M., Ding, H. J., Rees-Lee, P., \& Jensen, G. J. (2019, April). ETDB-Caltech: A blockchain-based distributed public database for electron tomography. PLoS One, 14(4), e0215531. Advance online publication. doi:10.1371/journal.pone.0215531 PMID:30986271

Palandini, M. (2018). Pharma Supply Blockchain Research Report: State of Blockchain Adoption on the Pharma Supply Chain. https://blockchain.ieee.org/standards/pharmasupply

Rowley, J. (2018). With at least $\$ 1.3$ billion invested globally in 2018, VC funding for blockchain blows past 2017 totals. TechCrunch. https://techcrunch.com/2018/05/20/with-at-least-1-3-billion-invested-globally-in-2018-vcfunding-for-blockchain-blows-past-2017-totals/

Script Drop Integrated Prescription Delivery. (2018). https://www.scriptdrop.co

Sharma, T., Banerjee, K., Mathur, S., \& Bali, V. (2020). Stress analysis using machine learning techniques. International Journal of Advanced Science and Technology, 29(3), 14654-14665. http://sersc.org/journals/ index.php/IJAST/article/view/31952

Skolnick, P. (2018). The opioid epidemic: Crisis and solutions. Annual Review of Pharmacology and Toxicology, 58(1), 143-159. doi:10.1146/annurev-pharmtox-010617-052534 PMID:28968188

Swati, V., \& Prasad, A. S. 2018. Application of blockchain technology in the travel industry. IEEE International Conference on Circuits and Systems in Digital Enterprise Technology, 1-5. doi:10.1109/ICCSDET.2018.8821095

Treleaven, P., Brown, R. G., \& Yang, D. (2017). Blockchain technology in finance. Computer, 50(9), 14-17. doi:10.1109/MC.2017.3571047

World Health Organisation. (2010). Growing Threat From Counterfeit Medicines. 10.2471/BLT.10.020410

Xia, Q., Sifah, E. B., Asamoah, K. O., Gao, J., Du, X., \& Guizani, M. (2017). MeDShare: Trust-Less medical data sharing among cloud service providers via blockchain. IEEE Access: Practical Innovations, Open Solutions, 5, 14757-14767. doi:10.1109/ACCESS.2017.2730843

Yadav, N., Banerjee, K., \& Bali, V. (2020). A survey on fatigue detection of workers using machine learning. International Journal of E-Health and Medical Communications, 11(3), 1-8. doi:10.4018/IJEHMC.2020070101

Yang, Z., Zheng, K., Yang, K., \& Leung, V. C. (2017). A Blockchain-based reputation system for data credibility assessment in vehicular networks. IEEE 28th Annual International Symposium on Personal, Indoor, and Mobile Radio Communications (PIMRC), 1-5. doi:10.1109/PIMRC.2017.8292724

Zīle, K. \& Renāte, S. (2018). Blockchain use cases and their feasibility. Applied Computer Systems, 12-20. $.10 .2478 /$ acss-2018-0002 
Vikram Bali is Professor and Head-Computer Science and Engineering Department at JSS Academy of Technical Education, Noida, India. He had graduated from REC, Kurukshetra - B. Tech (CSE), Post Graduation from NITTTR, Chandigarh - M.E (CSE) and doctorate (Ph.D) from Banasthali Vidyapith, Rajasthan. He has more than 20 years of rich academic experience. He has published more than 50 research papers in International Journals/Conferences and edited Books. He has authored Five textbooks. He has published Patent on Smart Dustbin- Sanitation \& Solid-Liquid Waste Management. He is on editorial and on the review panel of many International Journals. He is life time member of IEEE, Indian Society for Technical Education (ISTE), Computer Society of India (CSI) and Institution of Engineers (IE). He was Awarded Green Thinker Z-Distinguished Educator Award 2018 for remarkable contribution in the field of Computer Science and Engineering at 3rd International Convention on Interdisciplinary Research for Sustainable Development (IRSD) at Confederation of Indian Industry (CII), Chandigarh. He has also attended Faculty Enablement programme organised by Infosys and NASSCOM. He has been the member of board of studies of different Indian Universities and member of organizing committee for various National and International Seminars/Conferences. He is working on three sponsored research projects funded by TEQIP-3 and Unnat Bharat Abhiyaan. He has written books on Fundamental of "Cyber Security and Laws", "Software Engineering" and "Operating System". He is reviewer to many International Journals of repute like Inderscience and IGI Global. His research interest includes Software Engineering, Cyber Security, Automata Theory, CBSS and ERP.

Tejaswi Khanna, M.Tech. (YMCAUST, Faridabad), B.Tech. (KU, Kurukshetra), is a Ph.D. Scholar in Sharda University, Greater Noida. Currently serving as Assistant Professor in Computer Science and Engineering Department in JSS Academy of Technical Education, Noida, he is Member of IAENG and IRED. His research interest includes Computer Networks, Database Management Systems, Distributed System, Blockchain, Network Security and Social Networking. 